\title{
Segmentation of Noisy Binary Images Containing Irregular Shaped Objects using Genetic Algorithm
}

\author{
B. D. Phulpagar \\ Computer Engineering Department \\ P. E. S. Modern College of Engineering, \\ Pune, India.
}

\author{
R. S. Bichkar \\ Electronics and Telecommunication Department \\ G. H. Raisoni College of Engineering and \\ Management, Pune, India.
}

\begin{abstract}
Segmentation is one of the most important steps in image analysis. Image segmentation is the process of separating the foreground objects from the background. The earlier techniques use Genetic Algorithms (GAs) to separate the images containing regular, circular and elliptical-shaped objects from the background. The proposed technique uses the GA to segment the images containing irregular shaped objects. The Parallel Computing Genetic Algorithm (PCGA) implemented using Matlab PCT toolbox is also used to reduce computation time of image segmentation. The GA and PCGA are implemented using one-point, two-point and multiplepoint crossover operators. The proposed GA-based approach gives us good results for noisy images containing irregular shaped objects as well as circular or elliptical and rectangular objects. The results obtained give $94 \%$ to $99 \%$ segmentation accuracy for different types of noise (Poisson, salt and pepper, Gaussian and Speckle) and high noise levels (SNR ranging between $1.75 \mathrm{~dB}$ to $8.75 \mathrm{~dB}$ ). A significant speedup is obtained by using PCGA compared with the serial GA implementation.
\end{abstract}

\section{General Terms}

Image Processing, Image Segmentation.

\section{Keywords}

Genetic Algorithm, Image Segmentation, Parallel Computing.

\section{INTRODUCTION}

Generally, GA is used to search near optimal results based on the principles of natural selection and set of populations. GAs do not require domain specific knowledge in their search method. In every generation of GA, the generation of a new population of candidate solution depends on genetic operators like reproduction, crossover and mutation. Applications of GA in image processing area include image segmentation, image enhancement, image compression, watermarking, steganography, object recognition and feature selection.

Generally GA is a slow technique requiring long execution time to obtain near-optimal solutions. However, GA lends itself easily to parallel processing due to independence in the calculation of fitness of individuals. Thus, we can take the advantage of multi-core processors available on today's computers to substantially reduce the execution time and thereby explore possibilities of getting better solutions. The Parallel Computing Toolbox (PCT) of Matlab is a useful tool to implement such GAs on multi-core processor. The main advantage of this toolbox is to reduce the development time of PCGA for image segmentation with minimum changes in the existing algorithms. The PCT toolbox hides the complexities of inter-processor communication from the end user for the
PCGA. GA-based image segmentation has been used in different image analysis applications. Bichkar and Ray [1] have proposed a GA-based method for tomographic reconstruction of circular and elliptical objects. The proposed algorithm is used to correctly detect the number of circular and elliptical objects and to estimate object parameters, namely the location, size and shape in given images. Jiang, Yang and Fan [2] presented the technique for cell image segmentation using Parallel GA (PGA). It finds elliptically shaped cells in noisy images. Fan, Jiang and Evans [3] proposed a PGA-based approach for volumetric segmentation of brain images. It detects complex objects in the human brain i.e. the lateral ventricles. Jiang and Fan [4] have proposed method for 3D medical image analysis using PGA, which is used in model based segmentation and multimodality image registration. Nanda, Pande and Kanungo [5] have proposed PGA-based image segmentation using Markov random field model. This unsupervised algorithm works for synthetic and real images. Kanungo, Nanda and Ghosh [6] have proposed the method to obtain adaptive threshold for image segmentation under uneven lighting conditions using PGA. Kussener [7] used GA and PCGA approaches to automatically detect contours. It shows the significant speedup by using PCGA compared with the GA. Phulpagar and Kulkarni [8] have proposed a GA-based method for reconstruction of 2-D images containing regular-shaped objects. It is applicable only for synthetic images. Fakhri et. al. [9] has studied different techniques of parallel computing for image processing. Phulpagar and Bichkar [10] proposed modified GA-based approach for reconstruction of 2-D images containing circular and elliptical objects. In this approach, GA uses morphological operations at random from a set of operators.

In this paper, the GA and PCGA-based methods are implemented for reconstruction of 2-D images containing irregular-shaped objects for noisy input images. The results obtained using GA and PCGA show an improvement in classification accuracy as well as faster convergence.

\section{IMAGE SEGMENTATION USING GA}

GA is an efficient population-based search and global optimization method. An individual in GA population, called as a chromosome, is a finite-length binary string code corresponding to a solution of the problem under consideration. Each individual has a fitness value that is some measure of its closeness to the actual solution.

This paper presents use of GA for segmentation of binary images having irregular-shaped objects of a particular intensity embedded in uniform background of another intensity. The test images are corrupted by different types of noise (Poisson, salt and pepper, Gaussian and Speckle). The binary 2-D image containing irregular-shaped objects is formed by using two distinct gray levels. The SNR of 2-D 
images of size $M \times N$ is defined [10] as the ratio of average signal power to average noise power. SNR is mean-squared error measure and its unit is decibel $(\mathrm{dB})$.

$$
S N R(d B)=10 \log _{10} \frac{\left(\sum_{(i=1, j=1)}^{(M, N)} A(i, j)^{2}\right)}{\left(\sum_{(i=1, j=1)}^{(M, N)}(A(i, j)-B(i, j))^{2}\right)}
$$

Where, $A(i, j)$ denotes the $(i, j)^{\text {th }}$ pixel of the original image and $B(i, j)$ denotes the $(i, j)^{\text {th }}$ pixel of the noisy image.

Let the noisy image be represented as $I=\left[I_{1}, I_{2}, \ldots, I_{L}\right]$ and the chromosome of $i^{\text {th }}$ individual in GA population be represented as $P_{i}=\left[p_{\mathrm{i}, l}, p_{i, 2}, \ldots, p_{i, L}\right]$. The fitness of individual $P_{i}$ is calculated using the mean absolute difference between the corresponding pixels of image represented by $P_{i}$ and the noisy input image as

$\operatorname{fitness}\left(P_{i}\right)=\frac{1}{\left(1+\frac{1}{L} \sum_{j=1}^{L}\left|P_{i, j}-I_{j}\right|\right)}$

GA is an iterative method. The initial population of GA is randomly generated and the fitness of each individual is calculated. Then successive generations improve it through repetitive applications of genetic operators namely, selection, crossover and mutation until the desired solution obtained. The population size is kept constant over the entire GA run.

The selection function is implemented by using the concept of biased roulette wheel [14], in which each individual is assigned a roulette slot in proportion to its fitness.

Reproduction operators consist of three functions i.e. morphological operations, crossover and mutation. Morphological operations [11], [12], are used to obtain connectivity of an object in a solution binary image.

To identify irregular shaped objects contained in image, we have used morphological operators, selected at random among the following set: opening followed by closing, closing followed by opening, opening, closing, dilation and erosion The structuring element $(Q)$ is a $3 \times 3$ or $5 \times 5$ mask with an appropriate intensity structure. Also, each bit of structuring element is selected at random.

The crossover operator uses one-point, two-point and multipoint mechanisms. The crossover operator is applied with probability $p_{c}$ which is typically between 0.50 to 0.95 .

The mutation operator is implemented as a $3 \times 3$ median filter, where the central pixel in $n \times n$ window is replaced with the median of all the pixel values in the window.

The quality of solution in a GA run depends on the population size and the number of iterations performed, besides several other parameters. A large population obviously requires more computation. Hence, the multiple processor cores in a computer can be used effectively to reduce the total execution time and possibly obtained a better solution. This is achieved using PCT toolbox of Matlab. The PCGA-based method is implemented by using parfor loop construction of a PCT toolbox of Matlab.

PCGA implementation for IP tasks work with three types of data sets. coloum-wise, row-wise and block-wise. In the proposed method it works on block-wise data sets. For example, for a $64 \times 64$ pixels image, the PCGA works on total of 16 sub-blocks of size $16 \times 16$.

\section{RESULTS AND DISCUSSIONS}

In this work, we have used 2-D binary synthetic images of size $256 \times 256$ pixels containing irregular-shaped objects with two classes having pixel values 100 and 200 . The different shapes and number of objects are evolved in these test images. The salt and pepper noise is added with density of 0.10 to the original image shown in Figure 1 (a) to obtain noisy image with SNR of $19.14 \mathrm{~dB}$ shown in Figure 1 (b).

The proposed GA works with $16 \times 16$ sized parts of these images. Thus each individual in GA is encoded as a matrix of size $16 \times 16$.

The GA parameters are set as follows, population size of 150 , crossover probability in the range between 0.50 to 0.70 , number of generations $=80$. A morphological operator is selected at random among set of morphological operators and each bit of structuring element is selected at random.

Figure 1 (c) and (d) shows the results obtained using the proposed GA and the earlier GA implementation by authors [10] with multiple-point crossover operators for noisy image in Figure 1 (b). It may be observed that the objects boundaries are detected more accurately in image (c) compared to that in image (d). The pixel classification accuracy is $98 \%$ with the proposed GA technique compared to $97 \%$ with previous GA technique. Thus, the proposed GA provides improvement in quality of segmentation compared to previous GA for images containing irregular shaped objects.

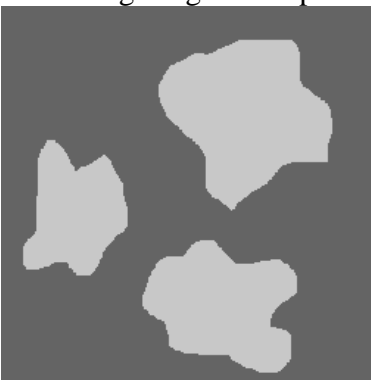

(a)

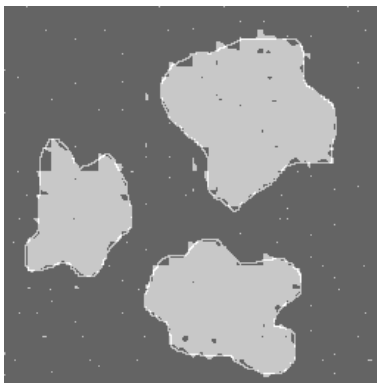

(c)

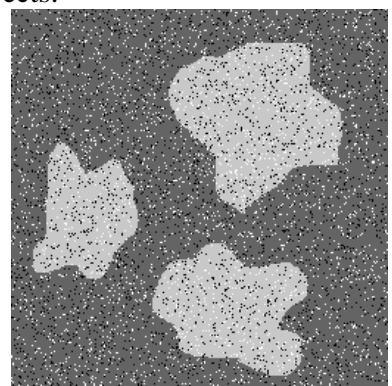

(b)

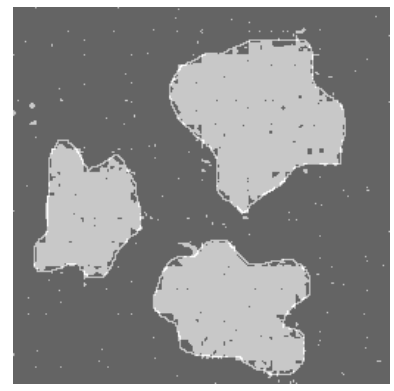

(d)
Figure 1: Segmentation results obtained by proposed GAbased approach for a noisy image (salt and pepper noise) containing irregular-shaped object. (a) Original image, (b) Noisy input image with salt and pepper noise with 19.14 dB SNR, (c) Result obtained by proposed GA-based approach and (d) Result obtained by authors previous GA-based approach [10].

Figure 2 shows convergence graphs of these GA runs where segmentation error is shown against the number of generations, for execution on a single processor core. It may be observed that the noise in the segmented image is high upto $5^{\text {th }}$ generations using previous GA method as compared to proposed GA method. The proposed GA shows a faster convergence compared to the earlier approach. 


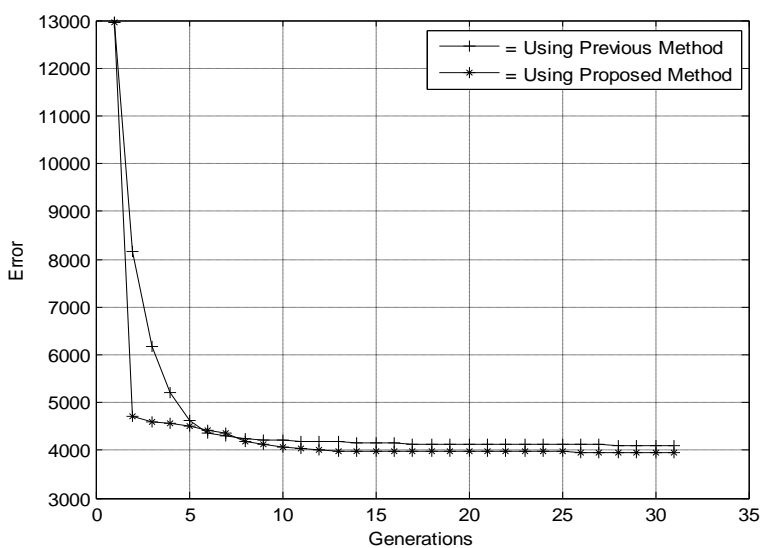

Figure 2: Convergence of the GA runs for results shown in Figure 1.

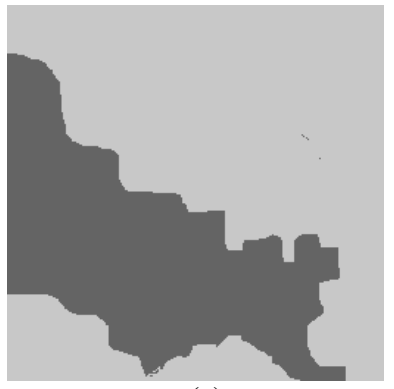

(a)

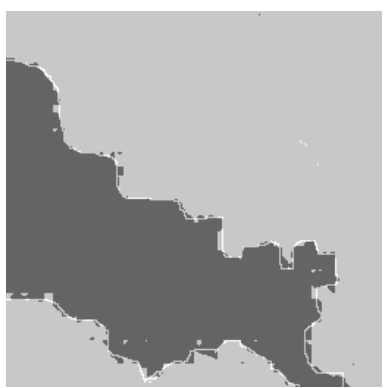

(c)

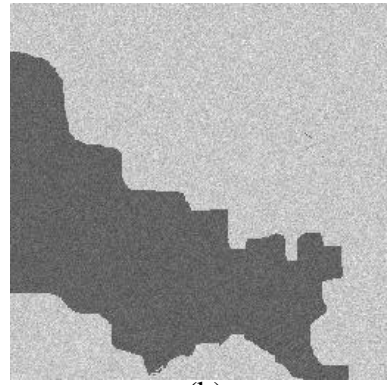

(b)

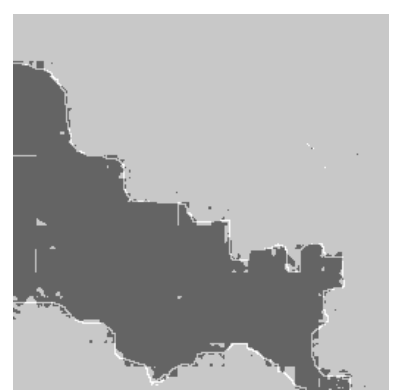

(d)
Figure 3: Segmentation results obtained by proposed GAbased approach for a noisy image (Poisson noise) containing irregular-shaped object. (a) Original image, (b) Noisy input image with Poisson noise with $4.78 \mathrm{~dB}$ SNR,

(c) Result obtained by proposed GA-based approach and (d) Result obtained by author's previous GA-based approach [10].

The proposed GA-based approach was also used to segment images containing Poisson noise. The most of the parameters of GA runs including population size, crossover and mutation rates etc. are the same in these runs as well.

Figure 3 (c) and (d) show the results obtained using proposed and previous GA-based approaches. Figure 3 (b) is an input image having Poisson noise with 4.78 dB SNR. Figure 3 (c) and (d) show the segmentation results having 99\% accuracy with the proposed GA and $98 \%$ accuracy with our earlier approach.

The proposed GA technique is also tested for different types of noises with higher noise density. The salt and pepper and Gaussian noise is added with density of 0.20 each and Speckle noise is added with density of 0.30 respectively to the original image to obtain noisy images shown in Figure $4(\mathrm{~b}-\mathrm{d})$ with SNR of $9 \mathrm{~dB}, 1.81 \mathrm{~dB}$ and $2.15 \mathrm{~dB}$ respectively. Figure 4 (a) is noisy image with SNR $5.39 \mathrm{~dB}$ obtained by adding Poisson noise to original image.

Figure 4: Second set of noisy test images corrupted with

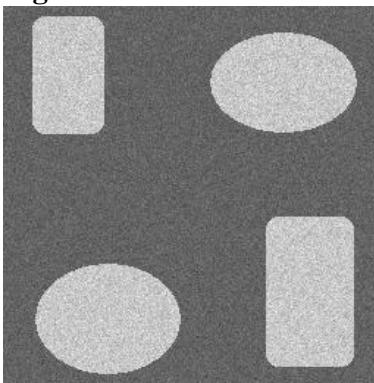

(a)

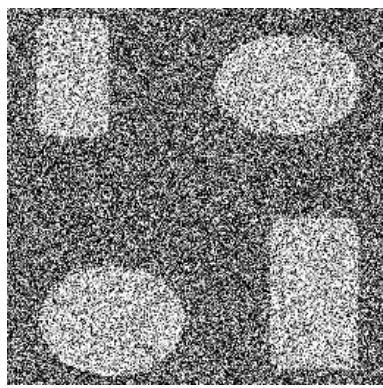

(c)

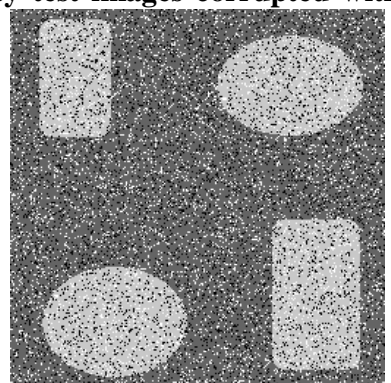

(b)

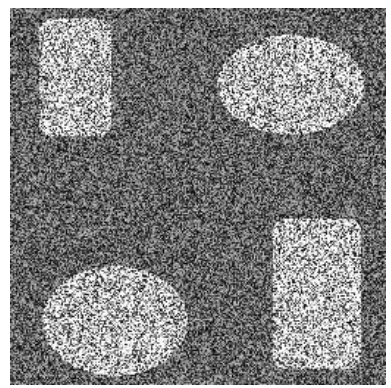

(d)
Poisson, salt and pepper, Gaussian and Speckle noise with $5.39 \mathrm{~dB}, 9 \mathrm{~dB}, 1.81 \mathrm{~dB}$ and $2.15 \mathrm{~dB}$ SNR respectively.

Next, Figure 5 shows the results obtained using proposed GA for noisy test images shown in Figure 4. It may be observed that very good segmentation is obtained after 75 iterations with pixel classification accuracy of $98 \%$ for both Poisson and salt and pepper noise as shown in Figure 5 (a) and (b). The results in Figure 5 (c) and (d) are for images having Gaussian and Speckle noises, the segmentation accuracy for which are $94 \%$ and $95 \%$ respectively. 
Volume 72-No.10, June2013

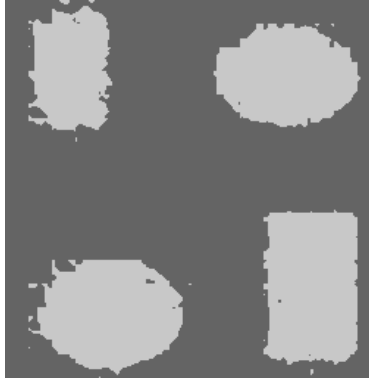

(a)

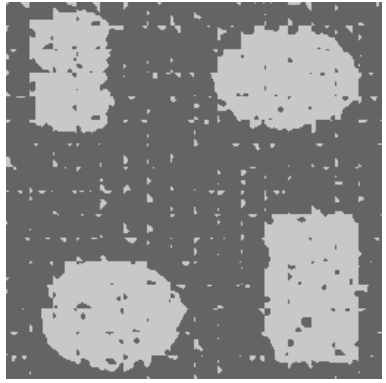

(c)

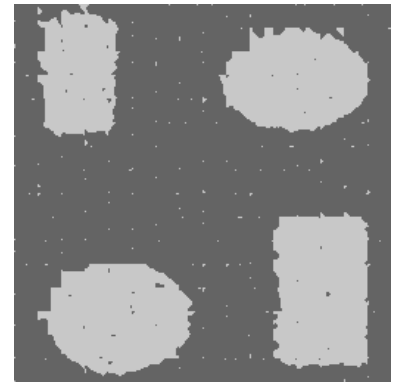

(b)

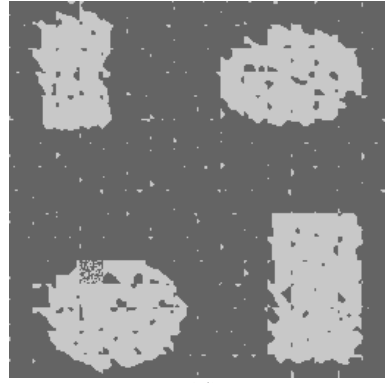

(d)
Figure 5: Results obtained by the proposed GA for test images shown in Figure 4.

The results presented so far have used GA with multiplepoint crossover operator. The single-point crossover operator was also found to be more or less equally effective for segmentation of noisy images shown in Figure 6.

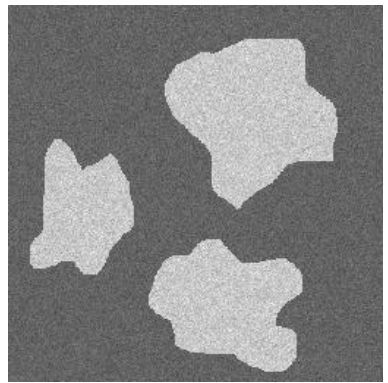

(a)

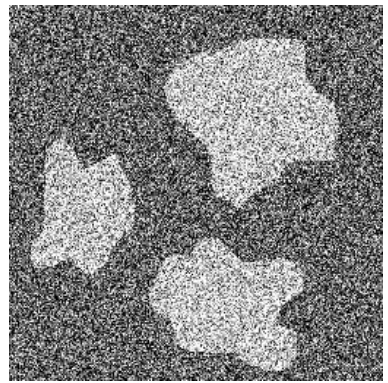

(c)

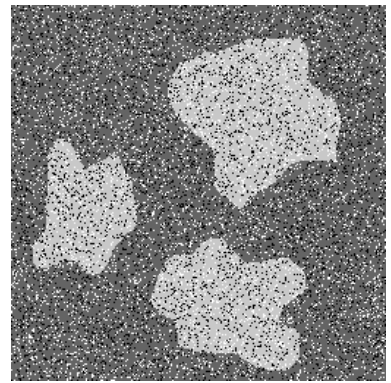

(b)

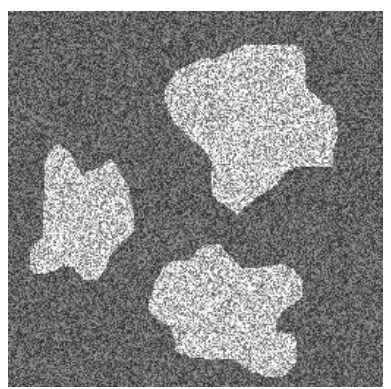

(d)
Figure 6: Noisy images with Poisson, salt and pepper, Gaussian and Speckle with $5.44 \mathrm{~dB}, 8.95 \mathrm{~dB}, 2 \mathrm{~dB}$ and 2.38 dB SNR respectively.

The results obtained using this GA with one-point crossover operator are shown in Figure 7, keeping other parameters same, where the images having Poisson, salt and pepper and Speckle noise have pixel classification accuracy of $98 \%$ and images containing Gaussian noise have classification accuracy of $96 \%$.

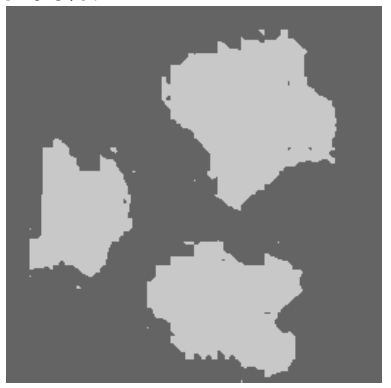

(a)

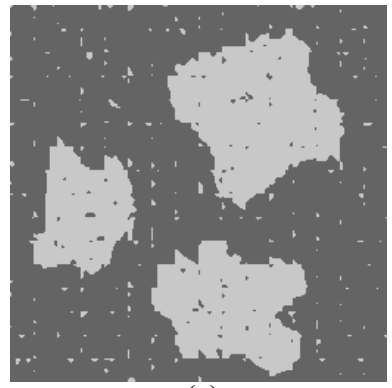

(c)

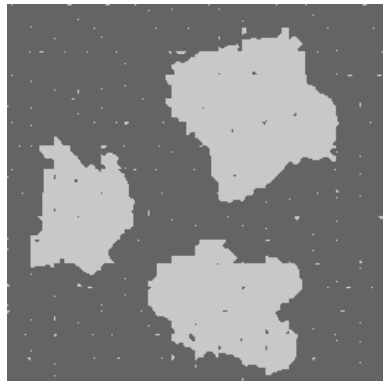

(b)

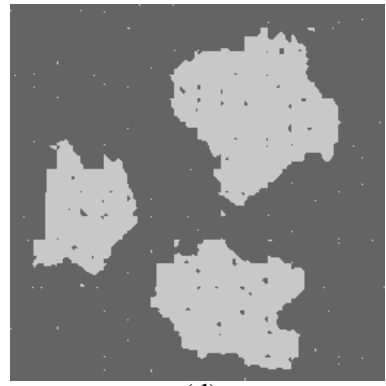

(d)
Figure 7: Segmentation results of GA with one-point crossover operator for images shown in Figure 6 (after 80 $^{\text {th }}$ iterations).

Further, we obtained segmentation results using two-point crossover operator and the results for noisy images in Figure 6 (c) and 6 (d) containing Gaussian and Speckle noise respectively are shown in Figure 8 (a) and 8 (b) respectively. The segmentation accuracy for these images is $96 \%$ and $97 \%$. This operator was also tested with noisy images shown in Figure 6 (a) and 6 (b) for which 98\% accuracy was obtained. Thus, two-point crossover operator also gives good results.

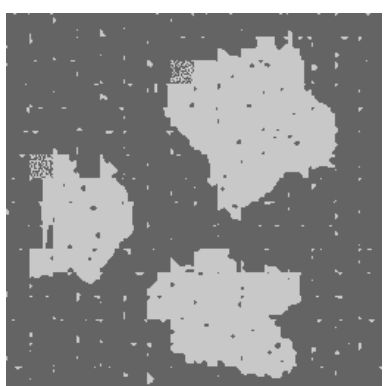

(c)

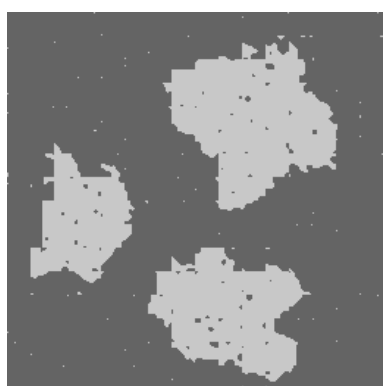

(d)
Figure 8: Results of GA with two-point crossover operator for the reconstruction of noisy images having Gaussian and Speckle noise shown in Figure 6 (c) and 6 (d) respectively.

Thus, the experimental result show that the GA with multiplepoint crossover method gives slightly better results than the two other crossover operators. The proposed GA is next applied to the larger synthetic images containing sharp and complicated irregular shaped objects of size $512 \times 512$ pixels. Figure $9(\mathrm{a}-\mathrm{d})$ shows the noisy test images having Poisson, salt and pepper, Gaussian and Speckle noise with SNR of 5.77 $\mathrm{dB}, 8.97 \mathrm{~dB}, 1.75 \mathrm{~dB}$ and $2.16 \mathrm{~dB}$ respectively. The segmentation accuracy of the results shown in Figure $10(\mathrm{a}-$ d) is $99 \%, 98 \%, 95 \%$ and $97 \%$. 


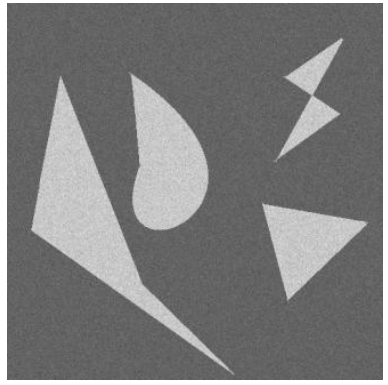

(a)

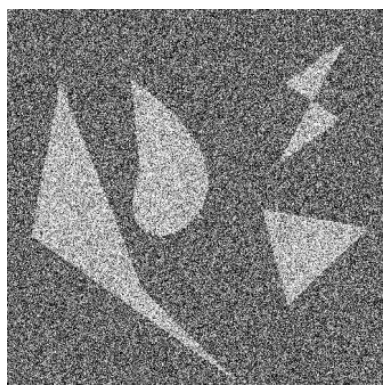

(c)

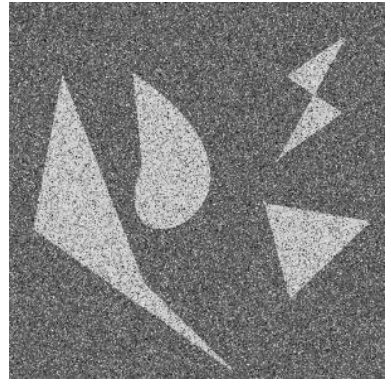

(b)

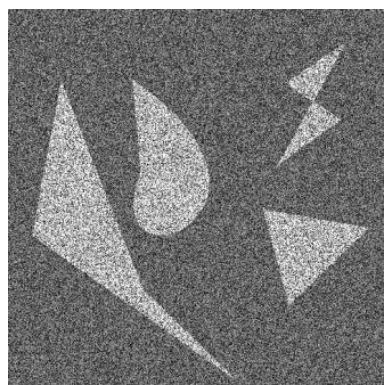

(d)
Figure 9: Larger-size noisy test images $(512 \times 512$ pixels $)$, (a) Poisson, (b) salt and pepper, (c) Gaussian and (d) Speckle images with $5.77 \mathrm{~dB}, 8.97 \mathrm{~dB}, 1.75 \mathrm{~dB}$ and $2.16 \mathrm{~dB}$ SNR respectively.

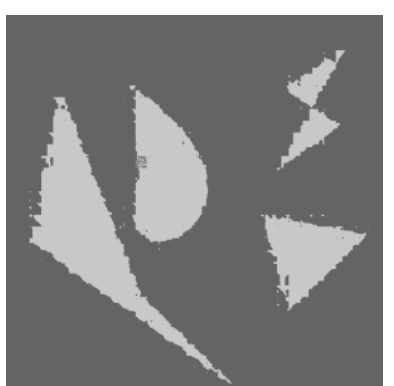

(a)

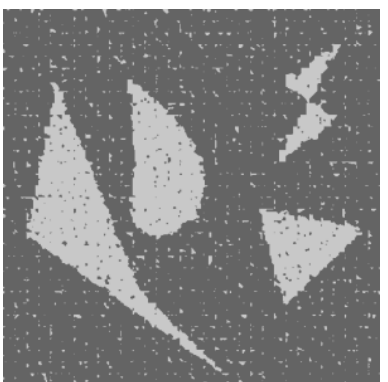

(c)

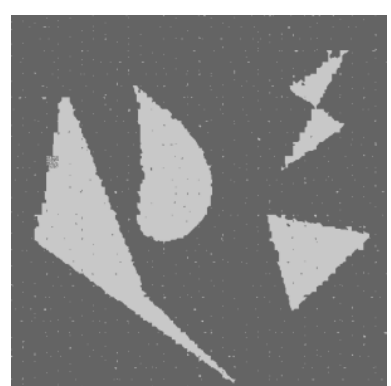

(b)

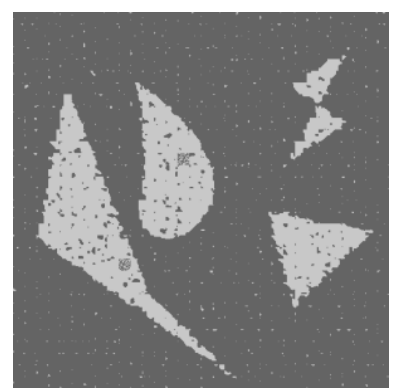

(d)
Figure 10: Segmentation results using proposed GA with multiple-point crossover for larger-sized noisy images shown in Figure 9.

The proposed GA is also tested implementation using onepoint and two-point crossover operators. It is an obvious from the results presented so far that the proposed GA-based approach is very powerful for image segmentation. However it requires large execution time. For example, while working on a machine having Intel i5-2450M CPU @2.50GH processor, 32-bit operating system and 4GB RAM, we required more than 80 mints for a GA run comprising of a population of 150 individuals executed for 80 generations (image size $256 \times 256$ pixels). We can greatly reduce the time required for obtaining these results using Parallel Computing GA, implemented using Parallel Computing Toolbox (PCT) of Matlab. Figure 11 shows the execution time required for segmentation of image of size $256 \times 256$ pixels on single core, two cores and four cores using GA multiple-point crossover. Figure 12 shows another execution time verses number of cores for larger image of size $512 \times 512$ pixels.

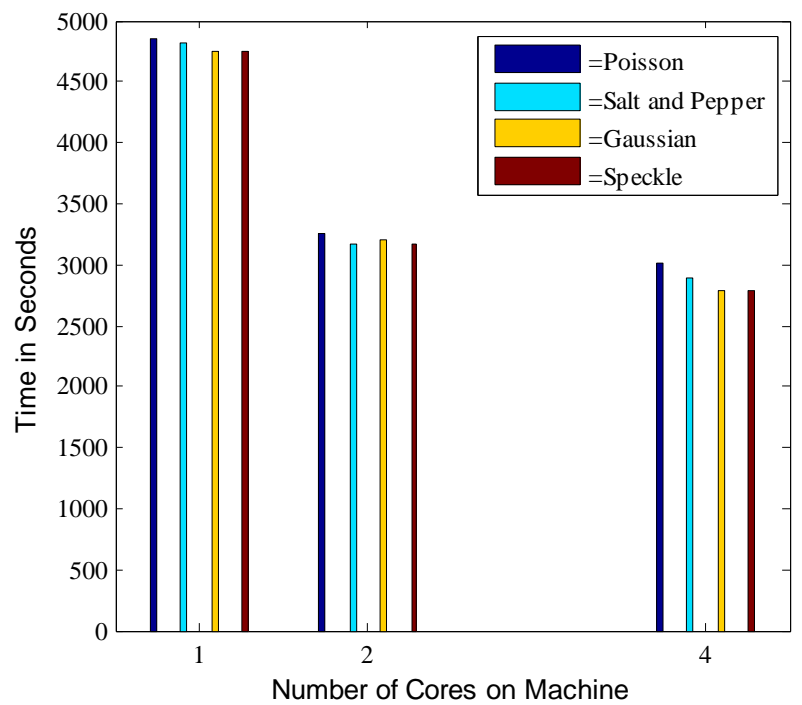

Figure 11: Execution time required for segmentation of $256 \times 256$ sized image using GA with multiple-point crossover.

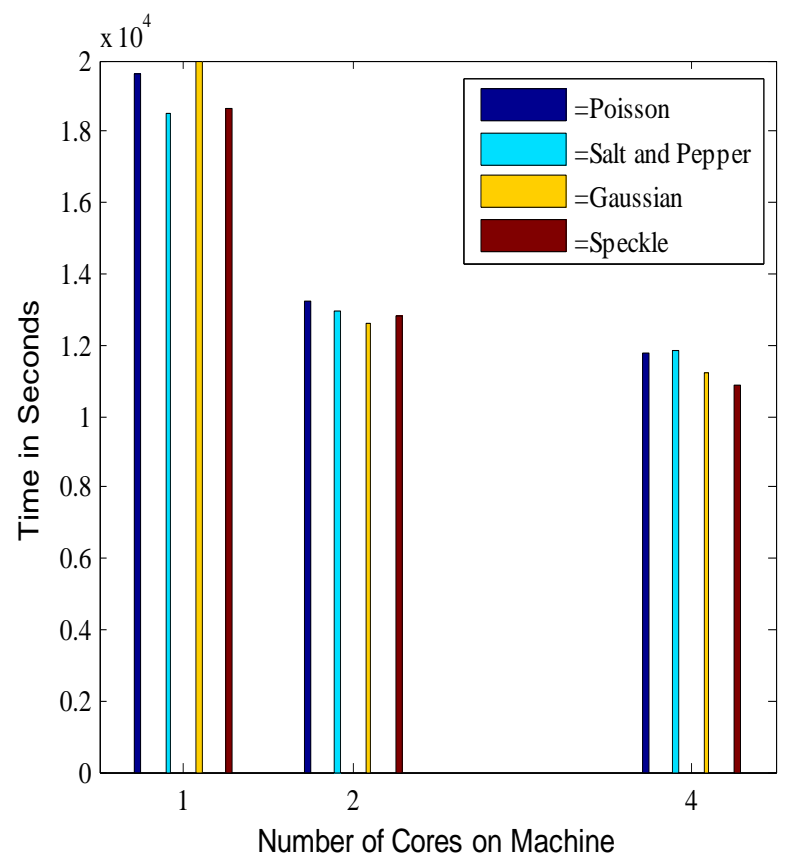

Figure 12: Execution time required for segmentation of $512 \times 512$ sized image using GA with multiple-point crossover. 
Table I shows the overall summary for image segmentation of 2-D binary images containing irregular shaped objects as well as circular or elliptical objects using GA and PCGA with multiple-point, one-point and two-point crossover operators.

Table I: Summary of segmentation results obtained using the proposed GA based approach.

\begin{tabular}{|c|c|c|c|c|c|c|c|c|c|c|c|c|c|c|}
\hline \multirow{3}{*}{$\begin{array}{l}\text { Sr. } \\
\text { No. }\end{array}$} & \multirow{3}{*}{$\begin{array}{c}\text { Figure } \\
\text { Number }\end{array}$} & \multirow{3}{*}{$\begin{array}{l}\text { Crossover } \\
\text { Operator }\end{array}$} & \multicolumn{12}{|c|}{ Segmented Results for Different Type of Noises } \\
\hline & & & \multicolumn{3}{|c|}{ Poisson Noise } & \multicolumn{3}{|c|}{ Salt and Pepper Noise } & \multicolumn{3}{|c|}{ Gaussian Noise } & \multicolumn{3}{|c|}{ Speckle Noise } \\
\hline & & & $\begin{array}{l}\text { SNR } \\
\text { in } \mathrm{dB}\end{array}$ & $\begin{array}{l}\text { Error } \\
\text { Pixels }\end{array}$ & $\%$ & $\begin{array}{l}\text { SNR } \\
\text { in } \mathrm{dB}\end{array}$ & $\begin{array}{l}\text { Error } \\
\text { Pixels }\end{array}$ & $\%$ & $\begin{array}{l}\text { SNR } \\
\text { in } \mathrm{dB}\end{array}$ & \begin{tabular}{c|} 
Error \\
Pixels
\end{tabular} & $\%$ & $\begin{array}{l}\text { SNR } \\
\text { in } \mathrm{dB}\end{array}$ & \begin{tabular}{l|} 
Error \\
Pixels
\end{tabular} & $\%$ \\
\hline \multirow{2}{*}{1} & \multirow[t]{2}{*}{ Figure $1(\mathrm{~b})$} & \multirow{5}{*}{ Multi-point } & & & & \multirow{2}{*}{19.14} & 1400 & 98 & & & & & & \\
\hline & & & & & & & $1780^{*}$ & $97^{*}$ & & & & & & \\
\hline \multirow{2}{*}{2} & \multirow{2}{*}{ Figure 3 (b) } & & \multirow{2}{*}{4.78} & 1019 & 98 & & & & & & & & & \\
\hline & & & & $1340^{*}$ & $97 *$ & & & & & & & & & \\
\hline 3 & $\begin{array}{c}\text { Figure } 4 \\
(a-d)\end{array}$ & & 5.39 & 1451 & 98 & 9 & 1595 & 98 & 1.67 & 3652 & 94 & 2.16 & 3304 & 95 \\
\hline 4 & $\begin{array}{c}\text { Figure } 6 \\
(\mathrm{a}-\mathrm{d})\end{array}$ & One-point & 5.44 & 1064 & 98 & 8.95 & 1425 & 98 & 2 & 2333 & 96 & 2.38 & 1599 & 98 \\
\hline 5 & $\begin{array}{c}\text { Figure } 6 \\
(\mathrm{a}-\mathrm{d})\end{array}$ & Two-point & 5.44 & 1482 & 98 & 8.95 & 1860 & 97 & 2 & 2599 & 96 & 2.38 & 2103 & 97 \\
\hline 6 & $\begin{array}{c}\text { Figure } 9 \\
(\mathrm{a}-\mathrm{d})\end{array}$ & Multi-point & 5.77 & 3676 & 99 & 8.97 & 4055 & 98 & 1.75 & 12463 & 95 & 2.16 & 8452 & 97 \\
\hline
\end{tabular}

* Author's earlier method

\section{CONCLUSION}

GA has been successfully employed for the image segmentation of 2-D binary images containing irregularshaped objects and corrupted with Poisson, salt and pepper, Gaussian and Speckle type of noises with varying densities. The proposed method gives good results as compared to previous method [10], for the images containing irregular shaped objects as well as circular or elliptical and rectangular objects. We achieved better segmentation results by choosing morphological operators at random and also selecting each bit of the structuring element at random. The results obtained with multiple-point crossover operator are slightly better as compared to GA with one-point and two-point crossover operators. The computation time of the large size of images is reduced by using the parallel computing genetic algorithm with minimum changes in the exiting GA algorithms. We can reduce execution time by $35 \%$ by using two cores and by $45 \%$ by using four cores.

\section{REFERENCES}

[1] R. S. Bichkar and A. K. Ray, "Tomographic Reconstruction of Circular and Elliptical Objects using Genetic Algorithm", IEEE Signal Processing Letters, Vol. 5 No. 10, PP. 248 - 251, October - 1998.

[2] T. Jiang, F. Yang and Y. Fan, "A Parallel Genetic Algorithm for Cell Image Segmentation", Elsevier Electronic Notes in Theoretical Computer Science, Vol. No. 46, PP. 1 - 11, 2001.

[3] Y. Fan, T. Jiang and D. Evans, "Volumetric Segmentation of Brain Images Using Parallel Genetic Algorithm", IEEE Transaction on Medical Imaging, Vol. 21, No. 8, PP. 904 - 909, August - 2002.

[4] T. Jiang and Y. Fan, "Parallel Genetic Algorithm for 3D Medical Image Analysis", IEEE International Conference on Systems, Man and Cybernetics, Vol. No. 6, PP. $1-6$, October - 2002.

[5] P. K. Nanda, S. Panda and P. Kanungo, "Parallel Genetic Algorithm based Textured Image Segmentation Using Markov Random Field Model”, National Conference on
Recent Advances in Power, Signal Processing and Control, PP. 161 - 166, November - 2004.

[6] P. Kanungo, P. Nanda and A. Ghosh, " Parallel Genetic Algorithm based Adaptive Thresholding for Image Segmentation under Uneven Lighting Conditions", IEEE International Conference on System, Man and Cybernetics, PP. 1904 - 1911, October - 2010.

[7] F. Kussener, "Active Contour: A Parallel Genetic Algorithm Approach", International Conference on Swarm Intelligence, PP. 1 - 9, June - 2011.

[8] B. D. Phulpagar and S. S. Kulkarni, "Image Segmentation using Genetic Algorithm for Four Gray Classes", IEEE International Conference on Energy, Automation and Signal, PP. $1-4$, December - 2011.

[9] A Fakhri, A Nasir and M. Nordin, "A Study of Image Processing in Agriculture Application under High Performance Computing Environment", IJCST, Vol. 3 Issue 8, PP. 16 - 24, August - 2012.

[10] B. D. Phulpagar and R. S. Bichkar, "Segmentation of Noisy Binary Images Containing Circular and Elliptical Objects using Genetic Algorithms", IJCA, Vol. 66, No. 22, PP. 1 - 7, March - 2013.

[11] R. C. Gonzalez, R. E. Woods, Digital Image Processing, Pearson, Delhi, 2008, $3{ }^{\text {rd }}$ Edition.

[12] A. K. Jain, Fundamental of Digital Image Processing, Pearson, Delhi, 1989, $2^{\text {nd }}$ Edition.

[13] E. Gose, R. C. Johnsonbaugh, Pattern Recognition and Image Analysis, Prentice Hall, Delhi, 2000, $2^{\text {nd }}$ Edition.

[14] D. E. Goldberg, Genetic Algorithm in Search, Optimization and Machine Learning, Pearson, Delhi, $2004,7^{\text {th }}$ Edition.

\section{BIOGRAPHIES}

Phulpagar B. D. is currently working as Assistant Professor in Computer Engineering Department at P. E. S. Modern College of Engineering Pune (India). He graduated from Govt. College of Engineering, Aurangabad affiliated with Dr. Babasaheb Ambedkar Marathwada University, Aurangabad and completed his postgraduate studies at Govt. College of Engineering Pune, affiliated to University of Pune. He is 
Pursuing Ph. D. in Computer Engineering at Shri Guru Gobind Singhji Institute of Engineering and Technology, Nanded, affiliated to Swami Ramanand Teerth Marathwada University Nanded (India).

Dr. Bichkar R. S. is currently working as a Professor in Department of E\&TC and Dean (R\&D), at G. H. Raisoni
College of Engineering and Management, Wagholi, Pune (India). $\mathrm{He}$ is $\mathrm{Ph}$. D. from Indian Institute of Technology Kharagpur (India). His areas of research include the application of Genetic Algorithms to Image Processing and Data Mining. 